\title{
mHealth App for Patient Self-Management of Chronic Kidney Disease Improves Renal Outcome: Pilot Study
}

Xuyong Chen ${ }^{1}, \mathrm{MD}$; Shasha Xie ${ }^{1}$, MBBS, MSc; Xiaojuan $\mathrm{Yu}^{2}$, MD; Zhibin Chen ${ }^{3}$, MD; Min Zhuo ${ }^{4}$, MD; Jiahua $\mathrm{Li}^{5}$, MBBS, PhD

\footnotetext{
${ }^{1}$ Beijing Kidney Health Technology Company Ltd, Beijing, China

${ }^{2}$ Renal Division, Department of Medicine, Peking University Health Science Center, Peking University First Hospital, Beijing, China

${ }^{3}$ Renal Division, Department of Medicine, Wenzhou Medical College, Yueqing Hospital, Yueqing, China

${ }^{4}$ Renal Division, Department of Medicine, Harvard Medical School, Beth Israel Deaconess Medical Center, Boston, MA, United States

${ }^{5}$ Renal Division, Department of Medicine, Harvard Medical School, Brigham and Women's Hospital, Boston, MA, United States
}

\author{
Corresponding Author: \\ Jiahua Li, MBBS, PhD \\ Renal Division, Department of Medicine \\ Harvard Medical School \\ Brigham and Women's Hospital \\ 75 Francis Street \\ Boston, MA, 02115 \\ United States \\ Phone: 7325500 \\ Email: jli54@bwh.harvard.edu
}

\begin{abstract}
Background: The prevalence of chronic kidney disease (CKD) is approximately 850 million worldwide and 120 million in China. Approximately $2 \%$ of the CKD population will progress to end-stage renal disease (ESRD) requiring renal replacement therapy or transplantation. The total health care expenditure on dialysis for the entire ESRD population in China is estimated to be 240 billion RMB per year. Using mobile health information technologies to conduct low-cost, large-scale, and personalized populational health interventions show a great promise.
\end{abstract}

Objective: In this pilot study, we assessed the feasibility and clinical effectiveness of a mobile application designed to improve patient's self-management of chronic kidney disease over a 3-month intervention with a pre-post design and a quasi-trial design.

Methods: Patients with CKD stage 1-3 and uncontrolled proteinuria (proteinuria $>1 \mathrm{~g}$ per day) were recruited. Eligible patients who were waitlisted served as the control. Patients in the experiment group were invited to install a mobile application known as Shen Shang Xian (Chinese pinyin for kidney online) for CKD self-management. The enrollment included a questionnaire for medical history and self-reported objective physical parameters and laboratory values. Each participant was assigned to one nephrologist who communicated with the patient on an ad-hoc basis. Blood pressure and laboratory test results were entered by the patients on a regular basis. The application has a built-in clinical decision algorithm to generate health recommendations to users based on one's data-entry. The application also sends various alerts to patient's nephrologist for timely interventions. Blood pressure, proteinuria, serum creatinine and eGFR were measured before and after the management period.

Results: Fifty-three patients were enrolled in the experimental group and 11 patients were in the control group. The average daily usage in minutes was $11.2(25 \%-75 \%$ quartile $[7.5,16])$ and the average of total physician-patient conversation was 116 (25\%-75\% quartile [51, 274]). There is a significant correlation between average daily usage and physician-patient conversation (R2=0.30, $P<.001)$. The starting eGFR was $\left.102 \mathrm{ml} / 1.73 \mathrm{~cm}^{2}(95 \% \mathrm{CI} 92-105]\right)$ in the experimental group and $118 \mathrm{ml} / 1.73 \mathrm{~cm}{ }^{2}$ (95\% CI 100-134]) in the control group ( $P=.04)$. The body mass index (BMI), blood pressure, and proteinuria had no statistical significance. At the end of the study, the mean change of proteinuria was $-1.39 \mathrm{~g}$ (95\% CI -2.07 to -0.72$])$ in the experimental group and $0.37 \mathrm{~g}(95 \% \mathrm{CI}-2.11-2.85])$ in the control group $(P=.14)$. After adjusted for ACEi/ARB use, the mean change of proteinuria was -1.46 vs 0.47 in the experimental group vs the control group respectively $(P=.16)$. The eGFR was not changed at the end of the study. There was no correlation between the average daily use and change of proteinuria.

Conclusions: Participants used the mobile app on a daily basis and communicated with the nephrologists for their CKD management. Patients who used the CKD self-management app exhibited a non-statistically significant trend of proteinuria 
reduction after 3 months. This pilot study was underpowered and the follow-up period was short. A larger retrospective controlled trial is needed to confirm the effectiveness of mHealth app in CKD self-management.

(iproc 2018;4(2):e11777) doi: $10.2196 / 11777$

\section{KEYWORDS}

self-management; chronic kidney disease

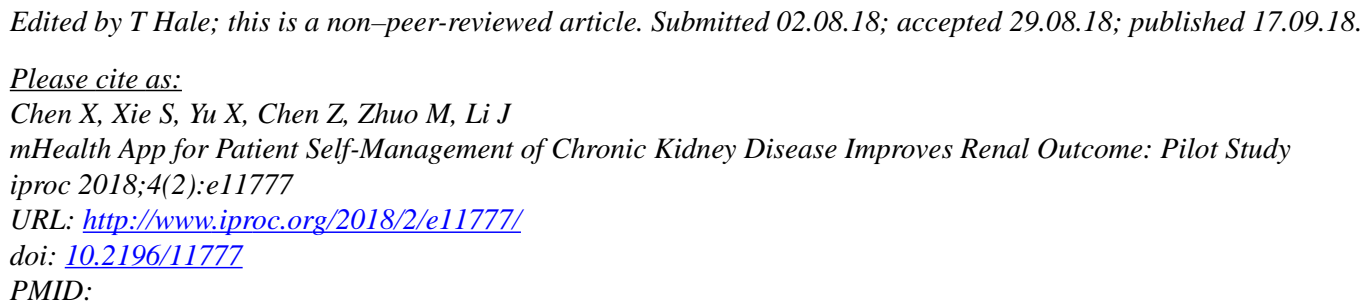

(CXuyong Chen, Shasha Xie, Xiaojuan Yu, Zhibin Chen, Min Zhuo, Jiahua Li. Originally published in Iproceedings (http://www.iproc.org), 17.09.2018. This is an open-access article distributed under the terms of the Creative Commons Attribution License (https://creativecommons.org/licenses/by/4.0/), which permits unrestricted use, distribution, and reproduction in any medium, provided the original work, first published in Iproceedings, is properly cited. The complete bibliographic information, a link to the original publication on http://www.iproc.org/, as well as this copyright and license information must be included. 